\title{
Introduction of Surface grinding equipment
}

\author{
Xuming Ye ${ }^{1 . a}$, Zhen Fang ${ }^{1 . b}$, Changsheng $\mathrm{Li}^{2}$ \\ 1.a Mechanical Engineering College ,Shenyang University Shenyang 110044,CHN \\ 1.b Mechanical Engineering College ,Shenyang University Shenyang 110044,CHN \\ ${ }^{2}$ Shen yang cheng sheng Industrial equipment factory, Shenyang 110044,CHN \\ b740609746@qq.com
}

Keywords: Surface; research and discussion; grinding.

Abstract. The outer surface of the continuous caster brass processing, for example, research and discussion process for the grinding of non-ferrous metal surfaces.

\section{Introduction}

With the continuous development of industrial technology, and some parts due to the need of the function and appearance etc, designed to special-shaped surface. Special-shaped parts refers to its characteristics different from general characteristics of the parts, and with two or more general feature of irregular parts. In this paper, we study is different from general cross section of special-shaped parts of rotary parts, this kind of special-shaped parts processing problem basically has: one is special-shaped parts clamping, conventional universal machine tool fixture positioning clamping complete stability; another one it is tool feeding needs to constantly adjust according to the surface of the special-shaped parts change. At present, based on the highly intelligent nc equipment for special-shaped parts surface processing to become each big mainstream mode of production machinery manufacturing enterprises, there are some machining enterprises more than using the traditional process of corresponding processing approach to the production of the type of pipeline. Both exist deficiencies, the former, equipment cost is higher; The latter, the production efficiency is low, the machining accuracy is restricted. To solve the above problems, " The soft can overcome the hard " with a new process of research topics, in" tool "of" soft "in the face of the special-shaped parts of irregular shape characteristics, complete the surface processing. Nonferrous metals has good plasticity, but in the process of processing prone to problems such as viscosity, the devolop tumor, burns, add more difficulties for the machining of the special-shaped parts surface. Therefore, in view of the non-ferrous metal special-shaped surface processing is the process of exploration is ongoing, seek effective and low cost processing project become a problem to be solved at present.

Continuous casting machine is in the process of industrial raw material to produce one of the most important equipment, demand is at a $4 \%$ growth rate increased year by year, its core components caster crystallizer copper tube is became a leading subsidiary material, become one of the heavy industry products.

Complex surfaces is difficult with conventional machine tools for processing, It has become the field of surface finishing a technical problem, and non-ferrous metals are soft, prone to plastic deformation, So can take some difficulty for shaped surface machining. When Caster mold brass production process, the outer surface have a large amount of oxide in the cold pushed molding process, in order to meet the need for subsequent processing, It must remove scale (refer with Figure 1). Each mold manufacturing enterprises, Because of lack of advanced equipment and consideration of costs and other factors, and Common processing equipment are Handheld grinder and Sandpaper. There are some problem that low productivity, Low productivity, Labor intensity and noise issues. To ensure accuracy, increase productivity and reduce labor intensity and so many problems, explore new technology for the processing of non-ferrous metal surfaces become technical problem to be solved at present. 


\section{A Process}

For shaped surface of parts, only to adapt to changes in its surface and to achieve flexible line go "knife" in order to solve the technical problems from now on. because Belt its own characteristics is called a flexible abrasive, It has a very high value in profiled surface processing field. As shown in Figure 1, mold brass blank piece is shaped copper or copper alloy surface by the plane and arc composed, using the wheel and alloy cutting tools for large surface oxide treatment is very difficult, but the flexible belt can be adapted to the surface shape of The complex changes continuously grinding.

As shown in Figure 2, this process is not only a conventional belt grinding, but also the belt attached to the surface of the gasbag, Airbag will rely belt is pressed against the surface of the workpiece, To the workpiece "Shaft" movement circumferentially along the curved shape, As a special "ring tool" rotary machining. Three airbags in the structure of Communicating vessel Conducted Unicom, inflated, each balloon internal pressure constant, It can ensure that any contact with the position of the belt and the workpiece surface contour is equal to normal pressure, positive pressure to ensure constant grinding. By the driving means and the feed means so that the "ring tool" to the workpiece rotation and linear motion of the base, so that the abrasive machining path shaped spiral form, the complete grinding the entire surface.

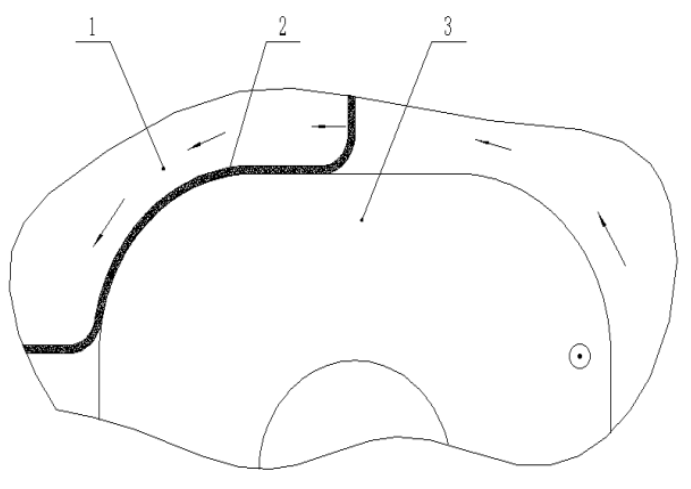

1-airbag 2- Belt 3-piece

Figure 2 A process schematic diagram

\section{Ring Tool}

Ring tool structure shown in Figure 3, Consist of intake pipe, tool holder, cylindrical roller, airbag spacer, airbag, belt and unicom airway. The intake pipe is mounted on an equipment rack, it can be up and down fine-tuned to ensure that the tool center and different sizes of work holding center line, adapt to different specifications mold brass blank processing. By Unicom airway making balloon positioned on the positioning frame of the balloon, and The balloon is synchronized with the rotation of the positioning frame, the airbag material requirements of flexibility, heat resistance temperature of 50 degrees Celsius, It has a high service life when deformation at high frequencies working conditions, Under the premise of choice to meet life as thin-walled balloon, and make the contact surface of the belt and the workpiece smoothing, before the work according to the workpiece dimensions of the airbag inflator modest. Airbag positioning groove belt rack power plant connected to the airbag and belt driven rotary. Choose a flexible belt substrate better adapt to the working status of the high-frequency distortion, the back belt in need of special treatment to the coefficient of friction with the air bag as small as possible to avoid excessive force airbag. For grinding front airbags should be inflated to the appropriate pressure values according to the workpiece sizes, specific numerical analysis to determine the need for experiments, the internal pressure of the airbag rely belt clamping force and the relative motion between the belt and the workpiece is applied to complete the grinding. 


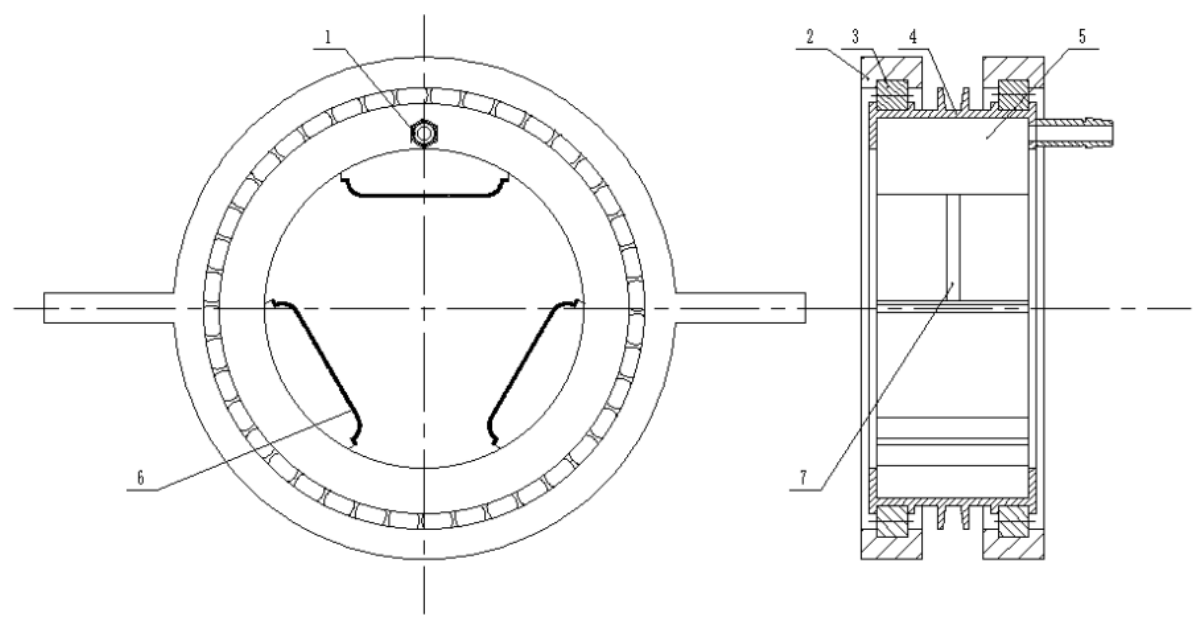

1-intake pipe 2-tool holder 3-Cylindrical Roller 4- airbag spacer 5-airbag 6- Belt 7- Unicom airway

Figure 3 Ring tool structure diagram Figure

Frame: by the steel welded together, it is the component parts of machine tool positioning base installation. Primary support and the effect of vibration reduction.

Feeding device: by the drive roller, bearing bracket, sprocket, roller chain, Zhang Jin device, etc. The axial feed mainly complete artifacts.

Conveyor roller: consists of metal roller and rubber roller, has good flexibility, ensure smooth workpiece feeding.

Guide frame: the guide roller, stents, adjusting screw, etc. Mainly to complete different specifications of artifacts, with the concentric ring tools. The main grinding head, grinding equipment, grinding main movement.

Grinding head rack: used for grinding head installation and adjustment of grinding head center height, make it adapt to the different specifications of grinding workpiece.

Motor: the main power source, through coupling transfer power to the grinding head, through reducer transfer power to feed device.
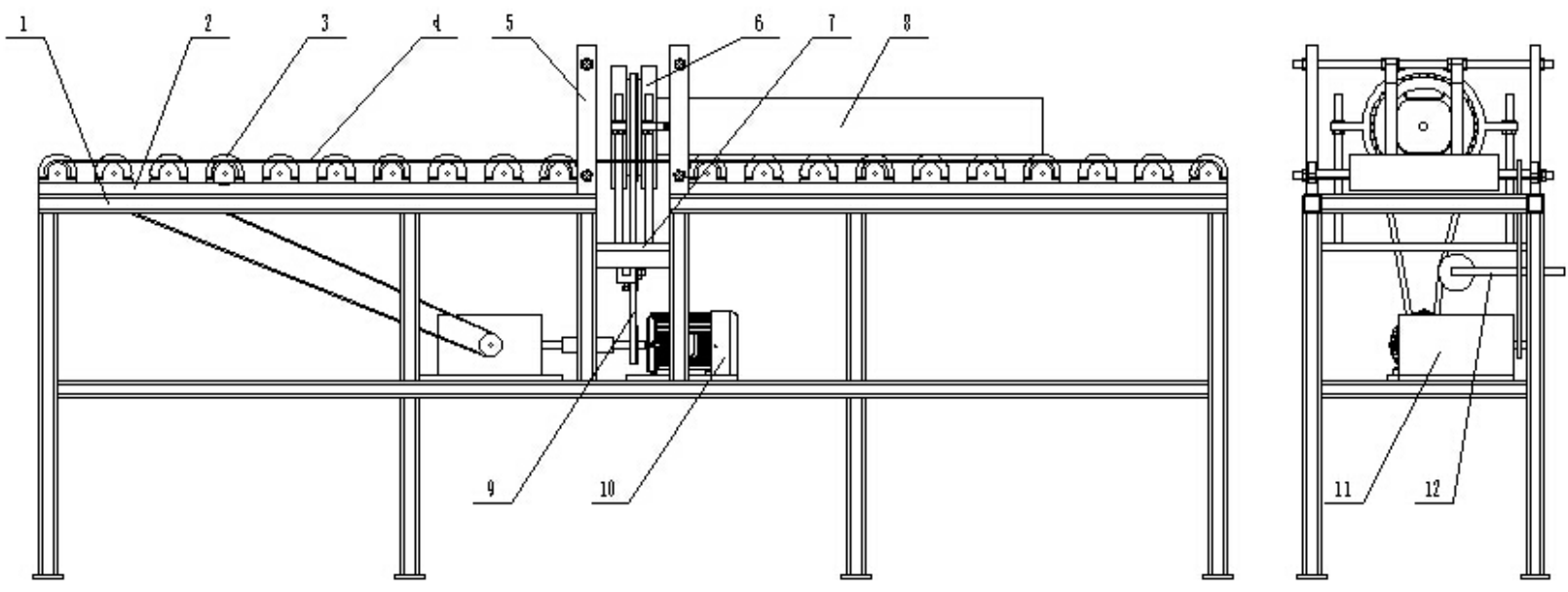

1 - rack 2 - feed unit 3 - conveying roller 4 - sleeve roller chain 5 - guide frame

6 - grinding head 7 - grinding head frame 8 - artifacts 9 - belt 10 - motor

Figure 4 general layout 


\section{Conclusion}

It is a flexible abrasive that belt has a high value in the surface grinding process. In this paper, continuous caster technical problems in the production process of enterprises brass blank faces were studied, introduced the "Ring Tool" This new grinding equipment, simple structure, low production cost and ease of application.

\section{References}

[1] Huxue Hong, Xu Fangjun glitch commonly used methods to analyze. J. technological innovation and application, 2014,06: 83.

[2] Sun miles, Shen Xin Gang, Fanjin Zhen, Wu Chun Bo, Geng Jinliang research lathe green belt polishing process. J. electrical engineering, 2014,02: 199-202.

[3] Fanru Yuan, Diou, Chenxin Xu flexible automatic polishing techniques. J. welding, 2014,06: 117-118.

[4] Huang Zhi, Huang belt grinding principle and its application. J. Metalworking (cold), 2008,24: 28-30.

[5] Zhu triumph, Chen Yanjun, Huang, Huang Zhi, Lin Yupei strong belt grinding research and design test machine. J. machinery manufacturing, 2006,03: 18-20. 